\section{P3.101 PREVALENCE OF HIV AND HCV AND HIV TRANSMISSION RELATED RISK FACTORS AMONG PERSONS WHO INJECT DRUG (PWID) IN BARNAUL, RUSSIA}

doi:10.1136/sextrans-2013-051184.0560

${ }^{1} \mathrm{~L}$ Sultanov, ${ }^{2} \mathrm{~L}$ Zohrabyan, ${ }^{3} \mathrm{E}$ Demianenko, ${ }^{4} \mathrm{~N}$ Vagaitseva, ${ }^{2} \mathrm{~J}$ Malkin, ${ }^{5}$ Toskin. ${ }^{\top}$ Center for AIDS and Infectious Disease Prevention of Altay Krai, Barnaul, Russian Federation; ${ }^{2}$ UNAIDS, Regional Support Team, ECA, Moscow, Russian Federation; ${ }^{3}$ Altay Kray Public Organization "AntiAIDS-Siberia", Batrnaul, Russian Federation; "Altay Kray Public Organization "AntiAIDS-Siberia", Moscow, Russian Federation; "Department of Reproductive Health \& Research (RHR) World Health Organization, Geneva, Switzerland

Background The annual numbers of registered HIV cases in Russia continue to grow. The epidemic is driven by use of contaminated illicit injection equipment. In 2006 HIV prevalence in Barnaul was $9 \%$ and Hep C 54\%. We sought to understand the epidemic trend and risk factors for HIV transmission in Barnaul.

Methods A cross-sectional survey PWID using RDS was conducted between August and October 2012 in Barnaul. PWID were recruited by 5 PWID seeds. Socio-behavioural, HIV and HCV results, and the proportion of previously undiagnosed HIV infections were calculated using RDSAT.

Results 500 PWID responded; they were predominantly: male (67.4\%, 95\% CI 62.3, 72.7), 30-39 years old $(54.7 \%$, 95\% CI 49.1, $60.1)$, single $(53.3 \%, 95 \%$ CI $48.0,59.4)$, unemployed $(67.3 \%, 95 \%$ $62.1,72.5)$, used alcohol in the last month $(81.6 \%, 95 \%$ CI 76.3 , 86.2). More than half of them injected in the last month $(55.9 \%$, $95 \%$ CI $50.4,61.5)$ from $\geq 2$ to $\leq 9(74.5 \%, 95 \%$ CI $66.9,84.0)$ times a day and $55.9 \%$ (95\% CI 52.1, 73.8) every day. Predominant drugs during the last month were heroin, marijuana (38.5\%, 95\% CI 26.0, 47.9) and desomorphine (19.0\%, 95\% CI 10.9, 23.8). 80.6\% of PIWD injected in a group with 1-5 injectors in the past one month $(95 \%$ CI 75.0, 88.3) with > 10 non-sterile injections $(59.1 \%, 95 \%$ CI 46.7 , $73.6)$ on average. $85.3 \%$ (95\% CI 80.5, 92.3) share paraphernalia. Prevalence of HIV was 30.3\% (95\% CI 25.3, 35.2) and Hep C $72.7 \%$ $(95 \%$ CI $67.5,77.7)$. Among those recently tested for HIV, $20 \%(95 \%$ CI 8.8, 28.8) were unaware they were HIV+.

Conclusions HIV prevalence more than tripled and Hep C has increased by $25 \%$ between 2006 and 2012. Increased prevalence may be attributed to high risk behaviours. Effective essential HIV prevention interventions must be introduced and/or scaled up rapidly to slow the epidemic.

\section{P3.102 HIV RISK IN PEOPLE WHO INJECT DIFFERENT DRUGS: SYSTEMATIC REVIEW AND META-ANALYSIS}

doi:10.1136/sextrans-2013-051184.0561

II Tavitian-Exley, 'M C Boily, ${ }^{2 P}$ Vickerman. 'Department of Infectious Disease Epidemiology, Imperial College, London, UK, London, UK; ${ }^{2}$ Social and Mathematical Epidemiology group and Centre for Research on Drugs and Health Behaviour, London School of Hygiene and Tropical Medicine, London, UK, London, UK

Objective To compare and understand how the risk of HIV infection in people who inject drugs (PWID) varies by substances and regions.

Methods A systematic review and meta-analysis was conducted. EMBASE and Medline were searched to identify cohort studies on HIV incidence in PWID injecting different drugs. HIV incidence rate ratio (IRR) was used to compare risk between drug injectors and non injectors, when possible, or study participants not injecting that substance, otherwise. Pooled estimates of unadjusted IRR (UIRR) were derived using random effects models. Sub-group analyses by substance and region were conducted and sources of UIRR variations explored.

Results Eleven cohort studies were included of 3,941 studies screened. HIV incidence by substance was reported for injecting cocaine ( 8 in Canada, America, Europe), amphetamine-type stimulants (ATS) (5 in Europe, Eastern Europe, Asia), heroin ( 9 in all regions), opiates and stimulants in (4 in America, Europe, Eastern Europe) and opiates and sedatives (4 in Europe, Asia). HIV risk for cocaine injectors was 3.5 times greater than non injectors (pooled $\mathrm{uIRR}=3.5,95 \% \mathrm{CI}: 2.9-4.3$ ) with increased sexual and injecting risk behaviours reported by cocaine-, compared to non-cocaine injectors in North America. HIV risk for heroin injectors was almost twice (pooled uIRR $=1.5,95 \%$ CI: 1.4-1.6) that of non- injectors in North America and four times (pooled UIRR $=4.0,95 \% \mathrm{CI}: 3.2-5.0$ ) in Europe and Asia, respectively. ATS yielded a pooled uIRR of 2.7 (95\% CI: 2.2-3.5) for Europe and Asia.

Conclusion Variations in the risk of HIV seroconversion found among injectors of different drugs suggest that specific substances influence different behavioural and biological infection pathways. Results support previous findings in Canada and America, that injecting cocaine carries the highest HIV risk. HIV risk across drugs by regions require further exploration. Substances injected should be considered in harm reduction and sexual risk reduction strengthened

\section{P3.103 SEXUAL ACT WITH MULTIPLE SEX PARTNERS IS AN UPCOMING THREAT FOR RAPID TRANSMISSION OF HIV AMONG PEOPLE WHO INJECT DRUGS (PWID) IN BANGLADESH}

doi:10.1136/sextrans-2013-051184.0562

A Bosu. Save the Children, Dhaka, Bangladesh

Introduction and Aims The 9th round national sero-surveillance data shows 5.3\% HIV prevalence among the PWIDs in Dhaka. Save the Children is committed to prevent HIV in Bangladesh where harm reduction is a major focus. At the beginning the project has conducted a study where one of the main focuses was to investigate the sexual behaviour and practise of the PWIDs.

Design and Methods The methodology used for this study was the Rapid Situation and Response Assessment by triangulation of both qualitative and quantitative method by collecting information from primary and secondary source. The data were collected through field observation, 790 one to one in-depth questionnaire, 42 Focus group discussions, 360 Key informant interviews and 14 cases studies.

Results Sixty percent (60\%) of PWIDs report to have had different sex partners on different occasions and of them 9 persons on average to have sex with. On average $13 \%$ of the PWIDs have experience of having sex in the anal route and only $21 \%$ of those had used condom. Only 44\% PWIDs reported that they had used condom in the last one year and consistent condom use is only $14 \%$.

Discussions and Conclusions Emphasizing safe sex behaviour programme with PWIDs is crucial to avert HIV transmission in Bangladesh.

\section{P3.104 THE SOCIAL ORGANISATION OF SEX WORK IN RURAL EASTERN ZIMBABWE AND ITS IMPLICATIONS FOR HIV INFECTION}

doi:10.1136/sextrans-2013-051184.0563

'J Elmes, ${ }^{2} \mathrm{~K}$ Nhongo, ${ }^{1} \mathrm{~T}$ Hallett, ${ }^{1} \mathrm{P}$ White, ${ }^{1} \mathrm{H}$ Ward, ' $\mathrm{G}$ Garnett, ${ }^{1} \mathrm{C}$ Nyamukapa, ${ }^{1} \mathrm{~S}$ Gregson. 'Imperial College, London, UK; ${ }^{2}$ Biomedical Research and Training Institute, Harare, Zimbabwe

Background Amid Zimbabwe's decline in overall HIV prevalence, the relative importance of key populations to ongoing transmission might be expected to change. We examine variation in the social organisation of sex work and assess the characteristics associated with HIV infection among sex workers (SW) in rural Zimbabwe.

Methods We collected and analysed cross-sectional data on 545 women who reported sex for payment - including cash-per-act and 
informal credit-based arrangements - and were recruited using snowball and location-based methods in October-December 2010. We used successive logistic regression models to explore determinants of HIV infection among women reporting venue-based sex work (e.g. bars - includes women recruited onsite) and women reporting non-venue based sex work.

Results HIV prevalence was significantly higher in venue SW compared to non-venue women $(55.5 \%$ vs. $41.9 \%$, p < 0.01$)$. Numbers of clients reported were low, but more venue SW reported 2 or more clients in the last fortnight ( $38 \%$ vs. $7.6 \%, p<0.01)$; consistent condom-use during commercial acts was similar ( $60 \%$ and $68 \%, p>0.1)$. More venue SW reported ever travelling away from where they live to sell sex $(27 \%$ vs. $12 \%, \mathrm{p}<0.01)$, initiated sex work earlier (age 25 vs. 28 years, $p<0.01$ ), and sold sex for longer ( 6 vs. 5 years, $p<0.05$ ). Factors associated with HIV infection were type of sex work (aOR: 2.2, 95\% CI: 1.3-3.8 vs. non-venue), and SW not requesting condom-use (aOR: 3.0, 95\% CI: 1.15-7.85), after controlling for basic demographic differences.

Conclusion Venue SW were more likely to be HIV-positive than non-venue women, although in both groups HIV prevalence was substantial, and consistent condom use low. High risk of HIV among women not requesting condom use highlights the importance to renew prevention efforts in this population. The results emphasise the need to understand SW more broadly.

\section{P3.105 USING RESPONDENT-DRIVEN SAMPLING TO ESTIMATE HIV AND SYPHILIS PREVALENCE AMONG FEMALE SEX WORKERS IN AGADIR, FES, RABAT AND TANGIER, MOROCCO}

doi:10.1136/sextrans-2013-051184.0564

${ }^{1} \mathrm{~L}$ Johnston, ${ }^{2} \mathrm{~A}$ Bennani, ${ }^{2} \mathrm{~A}$ Latifi, ${ }^{3} \mathrm{H}$ Oumzil, ${ }^{4} \mathrm{~B}$ El Omari, ${ }^{5} \mathrm{~F}$ El Rhoufrani, ${ }^{6} \mathrm{~L}$ Ouarsas, ${ }^{7} \mathrm{~K}$ Alami, ${ }^{7} \mathrm{H}$ El rhilani. 'University of California, San Francisco, CA, United States; 'Ministry of health, NAP, Rabat, Morocco; ${ }^{3}$ Ministry of health, INH, Rabat, Morocco; ${ }^{4}$ Unité de gestion du Fonds Mondial, Rabat, Morocco; ${ }^{5}$ Association de Lutte Contre le Sida, Rabat, Morocco; ${ }^{6}$ Association de Lutte Contre le Sida, Agadir, Morocco; 7 UNAIDS, Rabat, Morocco

Background Throughout the world, including in the Middle East and North Africa region, female sex workers (FSW) often have a disproportionately high prevalence of HIV infection and they, along with their clients, are considered a core group contributing to the transmission of HIV in many countries. FSW, a highly stigmatised, hard-to-reach and understudied population in Morocco were surveyed in four cities in Morocco, using Respondent-Driven Sampling.

Methods 372 FSW in Agadir, 359 in Fes, 392 in Rabat and 324 in Tangier were sampled over the course of six to eight weeks in December 2011 and January 2012. Eligible females reported exchanging penetrative (vaginal/anal) sex for money with more than one male client in the past six months, being 18 years or older, holding Moroccan nationality and working in the respective study location. Estimates were calculated using the multiplicity estimator in RDSAT V.6.0.

Results Most FSW in all four cities had no or low education, were separated, divorced or widowed and financially supporting adults and/or children. HIV seroprevalence in Agadir was 5.1\%, in Fes $1.8 \%$ and in Tangier $1.4 \%$. No one tested positive for HIV in Rabat. Syphilis infection in Agadir was $21.4 \%$, in Fes $18.8 \%$, in Rabat $13.9 \%$ and in Tanger $13.3 \%$.

Conclusions HIV among FSW in Morocco was lower than expected and confirms a concentrated epidemic in Agadir. Findings of syphilis infection among FSW may indicate infection with other sexually transmitted infections (STI) that were not tested in this survey and remain undetected. A scale up of programmes to provide targeted HIV outreach and services to FSW is essential to control the further spread of HIV and other STI in this population and to clients and other sexual partners.

\section{P3.106 POSSIBLE LINKS IN HIV INFECTION BETWEEN FEMALE SEX WORKERS (FSW) AND PEOPLE WHO INJECT DRUGS (PWID): FINDINGS FROM A RESPONDENT DRIVEN SAMPLING SURVEY IN DAR-ES-SALAAM, TANZANIA}

doi:10.1136/sextrans-2013-051184.0565

${ }^{1} \mathbf{N}$ - Makyao, ${ }^{2} \mathrm{~A}$ Kangolle, ${ }^{2} \mathrm{G}$ Arthur, ${ }^{3} \mathrm{M}$ Kazaura, ${ }^{4} \mathrm{~S}$ Welty, ${ }^{1} \mathrm{~B}$ Kilama, ${ }^{2} \mathrm{M}$ Kibona, ${ }^{1} \mathrm{~A}$ Ramadhani, ${ }^{3}$ S Kamazima. 'National AIDS Control Programme, Dar es Salaam, Tanzania; ${ }^{2}$ Centers for Disease Control and Prevention, Dar es Salaam, Tanzania; ${ }^{3}$ Muhimbili University of Health and Allied Sciences, Dar es Salaam, Tanzania; ${ }^{4}$ University of California San Francisco, California, CA, United States

Background Findings from studies done by McCurdy et al. have indicated that HIV prevalence may be as high as $42 \%$ among PWID in Dar es Salaam. Studies of PWID have also suggested overlaps between FSW and PWID populations. We investigated a Dar es Salaam FSW population to better understand possible linkages between these populations.

Method We conducted a cross-sectional study of FSW in 2010 using respondent-driven sampling (RDS). Participants completed questionnaires on socio-demographic variables, sexual and other risk behaviours; and had HIV testing. We used descriptive weighted statistics and logistic regression analysis to explore associations with HIV.

Results Among 537 FSW enrolled, 518 were tested for HIV giving $31.4 \%$ (95\% CI: $25.6,38.5)$ prevalence overall. In bivariate analysis, HIV prevalence was higher among FSW who suspected that partners injected drugs, $46.9 \%$ (95\% CI: $31.5,64.1)$ as compared to FSW who did not suspected that partners injected drugs $23.2 \%$ (95\% CI: 16.5, 29.4).

FSW rarely reported using drugs themselves $1.3 \%$ (95\% CI: 0.3 , 2.7), but often suspected their partners were PWID (49.1\%; $95 \% \mathrm{CI}$ : $43.8,55.5)$. In a multivariate model, adjusting for demographic and behavioural characteristics, the adjusted odds ratio (AOR) of HIV infection among women who suspected that their partners injected drugs was 2.41 95\% CI: 1.22, 4.80; (1.4 times greater) and was 0.08 times higher per additional year of sex work (AOR 1.08 95\% CI: 1.03, 1.1)

Conclusion These findings provide supportive evidence of the connexion between FSW and PWID populations in Dar es Salaam. Considering these apparent connexions between PWID and FSW, we encourage programmes working with these two key populations to work together to achieve enhanced prevention outcomes.

\section{P3.107 RESULTS OF THE FIRST HIV PREVALENCE AND RISK BEHAVIOUR STUDY AMONG FEMALE SEX WORKERS, BELIZE, 2012}

doi:10.1136/sextrans-2013-051184.0566

'B Alvarez Rodríguez, ${ }^{2} \mathrm{M}$ Manzanero, 'S Morales Miranda. 'Universidad del Valle de Guatemala, Guatemala, Guatemala; ${ }^{2}$ Ministry of Health, Belmopan, Belize

Background In Central America, studies have been conducted in several countries to provide baseline estimates of the prevalence of human immunodeficiency virus (HIV) infection and sexually transmitted infections (STIs), and to elucidate behaviours associated with HIV/STIs among Female Sex Workers (FSW). This study reports the results from the first behavioural and biological surveillance survey conducted in Belize.

Methods We used a census-based approach to recruit FSWs in four districts of Belize. FSW at least 18 years of age who reported vaginal or anal sex in exchange for money during the previous 12 months were invited to participate in the study. Participants were administered a questionnaire through audio computer-assisted self-interview and tested for HIV, syphilis, herpes simplex virus type 2 (HSV-2), Treponema pallidum (TP), Chlamydia trachomatis (CT), Neisseria gonorrhoeae (NG), Trichomonas vaginalis (TV), and 